\title{
A High Performance Robot Vision Algorithm Implemented in Python
}

\author{
Steven C. Colbert ${ }^{\S *}$, Gregor Franz $z^{\ddagger}$, Konrad Woellhaf ${ }^{\ddagger}$, Redwan Alqasemi ${ }^{\S}$, Rajiv Dubey ${ }^{\S}$
}

\begin{abstract}
A crucial behavior for assistive robots that operate in unstructured domestic settings is the ability to efficiently reconstruct the 3D geometry of novel objects at run time using no a priori knowledge of the object. This geometric information is critical for the robot to plan grasping and other manipulation maneuvers, and it would be impractical to employ database driven or other prior knowledge based schemes since the number and variety of objects that system may be tasked to manipulate are large.

We have developed a robot vision algorithm capable of reconstructing the 3D geometry of a novel object using only three images of the object captured from a monocular camera in an eye-in-hand configuration. The reconstructions are sufficiently accurate approximations such that the system can use the recovered model to plan grasping and manipulation maneuvers. The three images are captured from disparate locations and the object of interest segmented from the background and converted to a silhouette. The three silhouettes are used to approximate the surface of the object in the form of a point cloud. The accuracy of the approximation is then refined by regressing an 11 parameter superquadric to the cloud of points. The 11 parameters of the recovered superquadric then serve as the model of the object.

The entire system is implemented in Python and Python related projects. Image processing tasks are performed with NumPy arrays making use of Cython for performance critical tasks. Camera calibration and image segmentation utilize the Python bindings to the OpenCV library which are available in the scikits.image project. The non-linear constrained optimization uses the fmin___bfgs_b algorithm in scipy.optimize. The algorithm was first vetted in a simulation environment built on top of Enthought Traits and Mayavi.

The hardware implementation utilizes the Python OpenOPC project to communicate with and control a Kuka KR 6/2 six axis industrial manipulator. Images are captured via an Axis 207MW wireless network camera by issuing cgi requests to the camera with the urllib2 module. The image data is converted from JPEG to RGB raster format with the Python Imaging Library. The core algorithm runs as a server on a standalone machine and is accessed using the XML-RPC protocol. Not including the time required for the robot to capture the images, the entire reconstruction process is executed, on average, in 300 milliseconds.
\end{abstract}

Index Terms-computer vision, real-time, geometry, robotics

\section{Introduction}

Recently, the robotics and automation literature has seen an increase in research focus on the autonomous pose and shape estimation of general objects. The intent of these studies is that the pose and shape information of objects can be used to plan

* Corresponding author: sccolber@mail.usf.edu

$\S$ University of South Florida

+ University of Applied Sciences Ravensburg-Weingarten

Copyright (C) 2010 Steven C. Colbert et al. This is an open-access article distributed under the terms of the Creative Commons Attribution License, which permits unrestricted use, distribution, and reproduction in any medium, provided the original author and source are credited. grasping and manipulation maneuvers. In this context, such object recognition abilities have a plethora of applications that span multiple domains including, but not limited to: industrial automation, assistive devices, and rehabilitation robotics. Up to this point, a large portion of the research has focused on recognizing objects in which the system has some form of a priori knowledge; usually a $3 \mathrm{D}$ model or set of images of the object taken from various angles along with a database of information describing the objects. Recent examples of work in this area can be found in eg. [Kim09], [Effendi08], [Schlemmer07], [Liefhebber07], [Kragic05].

We approach this problem with the goal that the system need not have any prior knowledge of the object it wishes to manipulate. In the context of assistive or service robotics, requiring such $3 \mathrm{D}$ models or a database of information for every possible object would be prohibitively tedious and time consuming, thus severely limiting its usefulness and applicability. In order to achieve this goal, we attempt to describe generic objects in a bulk fashion. That is, to autonomously model an object's actual physical form at run time with a simplified shape that is an approximation; one which is also sufficiently accurate to allow for the planning and execution of grasping maneuvers. In our previous works [Colbert10_1], [Colbert10_2], we describe in detail the development of an algorithm that accomplishes just this. Only a brief overview of that theoretical work is presented here. Rather, the majority of this paper focuses on the implementation of that algorithm on an industrial manipulator and the accuracy of the reconstruction that results.

The paper progresses as follows: Section 2 provides the high level overview of the algorithm with some diagrams and a stepby-step visual example to ease conceptual understanding, Section 3 describes the software implementation of the algorithm, Section 4 describes the robotic hardware implementation to include networking and control, Section 5 elaborates on the testing and overall accuracy of the platform and algorithm under real-world conditions. We round out the paper with some conclusions in Section 6.

\section{Algorithm Overview}

This section provides a high-level overview of the theory behind our object reconstruction algorithm. No equations are presented. Rather the algorithm is explained qualitatively and the interested reader is directed to one of our previous works that develop the theory in detail: [Colbert10_1], [Colbert10_2]. 


\subsection{Shape from Silhouettes}

The first phase or our algorithm generates a rough approximation to the surface of an object using a method that falls under the category of shape from silhouettes. Algorithms of this class use a number of silhouettes of an object of interest captured from various vantage points and, by back-projecting the visual cones and finding their union, reconstruct the geometry of the object. As the number of available silhouettes increases to infinity, the reconstruction converges on the visual hull of the object [Laurentini94]. That is, the reconstruction will converge to the true shape of the object, minus any concavities. The method by which the visual cones are back projected varies from algorithm to algorithm, but most have typically used a variation of voxel coloring or space carving [Dyer01]. Our method is a modified version of a recently introduced new method of shape from silhouettes [Lippiello09]. Our modification to this algorithm utilizes projective geometry to eliminate the iteration step required in the original algorithm. The result is a shape from silhouettes algorithm that is conceptually easier to understand and computationally more efficient than historical methods.

Our algorithm begins by capturing three images of the object of interest from three disparate locations, and segmenting the object from the background. The segmented object is then converted to a silhouette. Then, using these silhouettes along with the known camera parameters, the $3 \mathrm{D}$ centroid of the object of interest is estimated. Along with the centroid, we estimate a radius of the object, which we define as a distance from the estimated centroid that would define the radius of a sphere that would fully encompass the object. Once this centroid and radius are determined, a virtual sphere of points can be constructed which fully encompasses the object. For each of the points in the sphere, the point is projected into the silhouette image and tested for intersection. If the point intersects the silhouette, nothing is done. However, if the point does not intersect the silhouette, its position in 3-space is modified such that its projected location in the image lies on the boundary of the silhouette. When this process is repeated for each silhouette, the resulting set of points will approximate the surface of the object. The geometry can be described with the following procedure and associated graphic:

1) Let the center of the camera be $\mathbf{c}_{0}$.

2) Let the center of the sphere be $\mathbf{x}_{0}$.

3) Let $\mathbf{x}_{i}$ be any point in the sphere other than $\mathbf{x}_{0}$.

4) Let $\mathbf{x}_{i_{n e w}}$ be the updated position of point $\mathbf{x}_{i}$.

5) Let the projection of the center of the sphere into the image be $\mathbf{x}_{0}^{\prime}$.

6) Then, for each point $\mathbf{x}_{i}$ :

a) Project $\mathbf{x}_{i}$ into the silhouette image to get $\mathbf{x}_{i}^{\prime}$.

b) If $\mathbf{x}_{i}^{\prime}$ does not intersect the silhouette:

i) Find the pixel point $\mathbf{p}^{\prime}$ that lies on the edge of the silhouette along the line segment $\mathbf{x}_{i}^{\prime} \mathbf{x}_{0}^{\prime}$.

ii) Reproject $\mathbf{p}^{\prime}$ into $\mathbb{R}^{3}$ to get the point $\mathbf{p}$.

iii) Let the line $\mathbf{c}_{0} \mathbf{p}$ be $\mathbf{L}_{1}$.

iv) Let the line $\mathbf{x}_{0} \mathbf{x}_{i}$ be $\mathbf{L}_{2}$.

v) Let $\mathbf{x}_{i_{\text {new }}}$ be the point of intersection of lines $\mathbf{L}_{1}$ and $\mathbf{L}_{2}$.

7) Repeat steps 2-6 for each silhouette image.

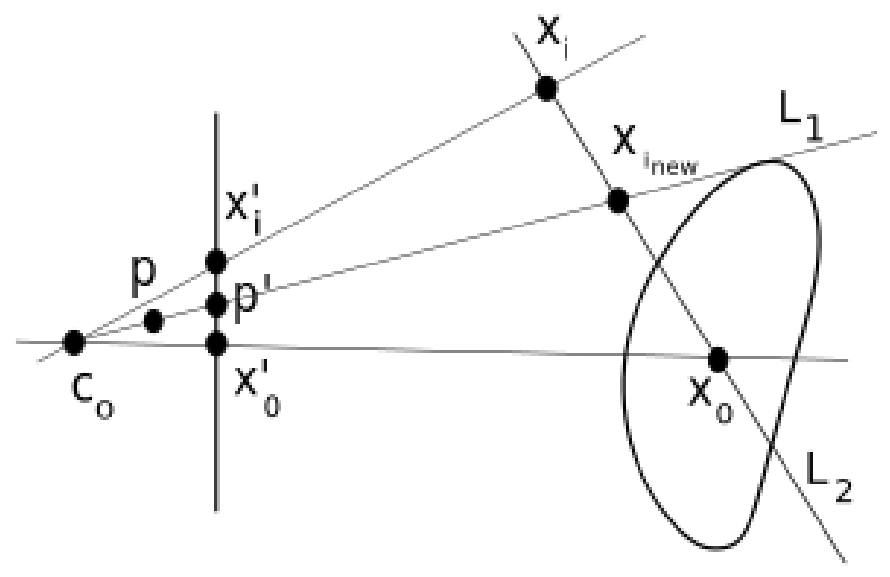

Fig. 1: The geometry of point $\mathbf{x}_{i_{n e w}}$, which is the intersection of lines $\mathbf{L}_{1}$ and $\mathbf{L}_{2}$. The line $\mathbf{L}_{2}$ is defined by known points $\mathbf{x}_{i}$ and $\mathbf{x}_{0}$. The line $\mathbf{L}_{1}$ is defined by point $\mathbf{c}_{0}$, which is the camera center, and point $\mathbf{p}$, which is the reprojection of the image point $\mathbf{p}^{\prime}$ into $\mathbb{R}^{3}$.

\subsection{Superquadrics}

The resulting set of points will, in general, be only a rough approximation of the surface of the object of interest. As previously mentioned, as the number of captured images becomes large, this approximation will become ever more accurate, but at the expense of increasingly long computation times. Our aim is to achieve usable results with a minimum number of images. To achieve a more accurate representation of the object using just three images, we fit a superquadric to the set of points which approximate the surface in such a manner that the superquadric largely rejects disturbances due to perspective projection effects and localized noise. The fitted superquadric then serves as a parametrized description of the object which encodes its position, orientation, shape, and size.

Our fitting routine is based on the methods proposed in [Jaklic00], whose work on superquadrics is authoritative. We made a modification to their cost function which heavily penalizes points lying inside the boundaries of the superquadric. This modification has the effect of forcing the fitting routine to ignore disturbances caused by perspective projection effects. For a few number of images, these disturbances can be large, and thus this modification is crucial to achieving a satisfactory reconstruction with only three images.

The reconstruction of a simulated shape is shown in the following figure. From the figure, it is clear that the fitted superquadric provides a substantially better approximation to the original shape than what can be achieved from the point cloud alone, when only three images of the object are available.

\section{Software Implementation}

The algorithm was developed and implemented entirely in Python. Images take the form of NumPy arrays with FOR loop dependent geometric image calculations performed in Cython. The Cython bindings to the OpenCV library (available in the scikits.image project) were used to build up the image segmentation routine. The fmin_1_bfgs_b non-linear constrained optimization routine (available in SciPy) was adopted for purposes of finding the best fitting superquadric for the point cloud. The gradient of the 

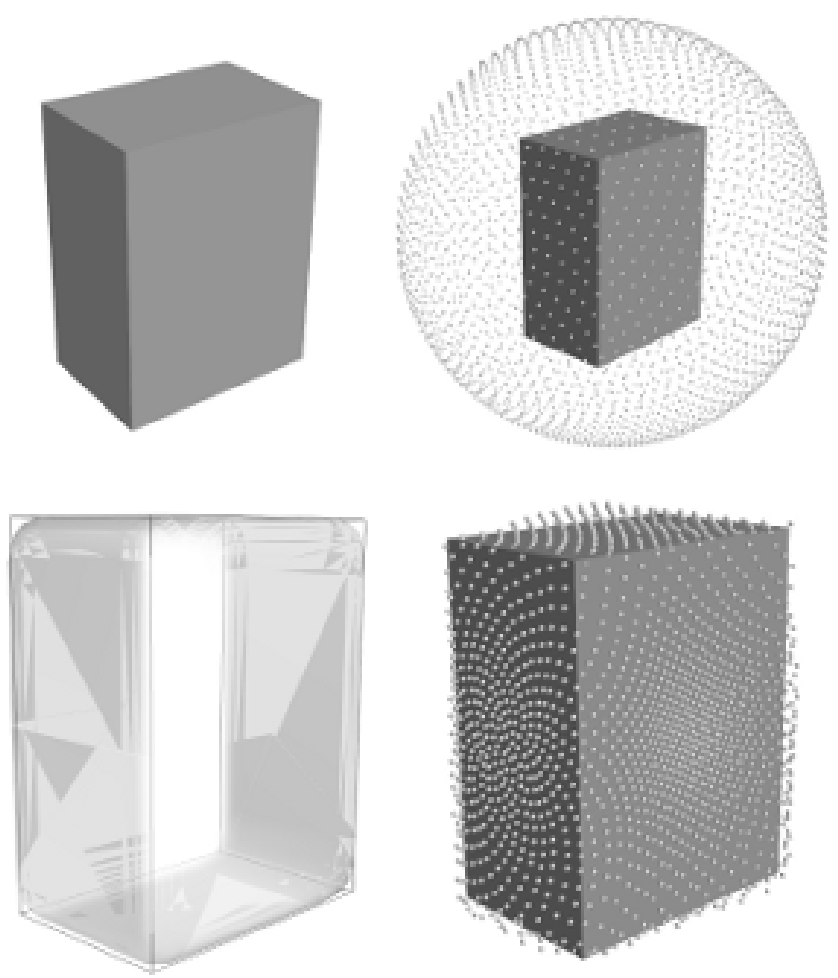

Fig. 2: A simulated reconstruction. Clockwise from upper left: (1) The original shape. (2) The generated sphere of points. (3) The point cloud after the points have been shrunk to the silhouette boundaries. Error due to perspective projection is clearly seen. (4) The superquadric that was fit to the point cloud. Original shape shown as a wire frame. Notice the ability of the superquadric to ignore the perspective projection error.

superquadric function (a hefty 296 SLOC) was implemented in Cython.

This software stack has proven to be quite performant. The average reconstruction time takes approximately 300 milliseconds. This includes image segmentation times but obviously does not include the time to actually capture the images. Compare this to the time taken for the reconstruction in [Yamazaki08] where a reconstruction using over 100 images required $~ 100$ seconds of processing time for an equivalent accuracy.

A simulation environment was also developed in concert with the algorithm for testing purposes. The environment uses Mayavi as a rendering engine and TraitsUI for the GUI. The environment allows simulating a number of various shapes and modifying their parameters in real-time. It also allows the images of the object to be captured from any position. Once the images are captured, the simulator then performs the reconstruction and displays the recovered superquadric as an overlay on the current shape. The computed accuracy of the reconstruction, based on the recovered superquadric parameters versus the known ground truth, is shown in a sidebar. Various intermediate stages of the reconstruction process are also stored as hidden layers for debugging purposes. These layers can be turned on after the reconstruction via dialog options. All of the reconstruction images in this text were generated with either the simulator or the underlying Mayavi engine. A screenshot of the simulator is shown below.

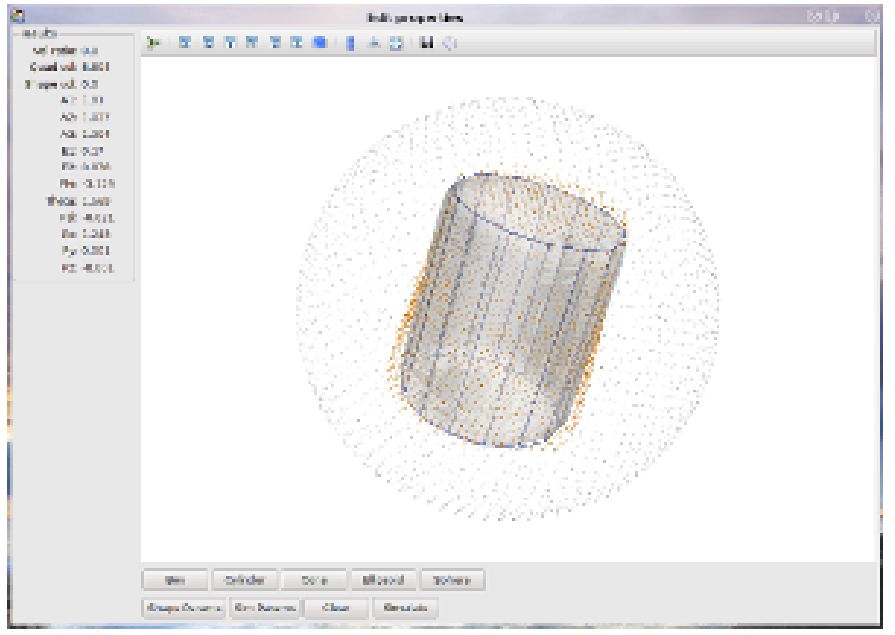

Fig. 3: A screenshot of the simulator which is built on Mayavi and TraitsUI.

\section{Hardware Implementation}

The implementation hardware consists of three main entities: the robotic manipulator which performs the required motions, the camera to capture the images, and the network which consists of the various components responsible for controlling the robot, the camera, and performing the actual object reconstruction computations.

It is desired to have these various systems interconnected in the most decoupled and hardware/operating system agnostic manner in order to facilitate software reuse on and with other platforms, robots, and cameras. Thus, portability was a chief goal behind the system design. The following sections describe each subsystem component in detail.

\subsection{Robot}

The robotic arm used for testing is a KUKA KR6/2, manufactured by KUKA Roboter GmbH. It is a six axis, low payload, industrial manipulator with high accuracy and a repeatability of $<0.1 \mathrm{~mm}$. It's smaller size (though still too large for use on a mobile platform) and large workspace makes it well suited for laboratory use and a wide range of experiments. The robot setup, including the camera described in Section 4.2 is shown in the following figure.

The KUKA control software provides a proprietary user interface environment developed in Windows XP Embedded, which in turn runs atop the real time VxWorks operating system. The platform provides a programming interface to the robot utilizing the proprietary KUKA Robot Language (KRL) as well as an OPC server that allows for connections from outside computers and the reading and writing of OLE system variables. As KRL does not provide facilities for communicating with outside processes or computers, the OPC server connection was used in conjunction with a simple KRL program to export control to an outside machine. The details of this are delayed until Section 4.3.

\subsection{Camera}

The camera used for image acquisition is an Axis 207MW wireless network camera. It is relatively inexpensive and has megapixel resolution. The main beneficial feature of the camera is that it contains a built in HTTP web server with support for acquiring images via CGI requests. This means that the camera can be used 


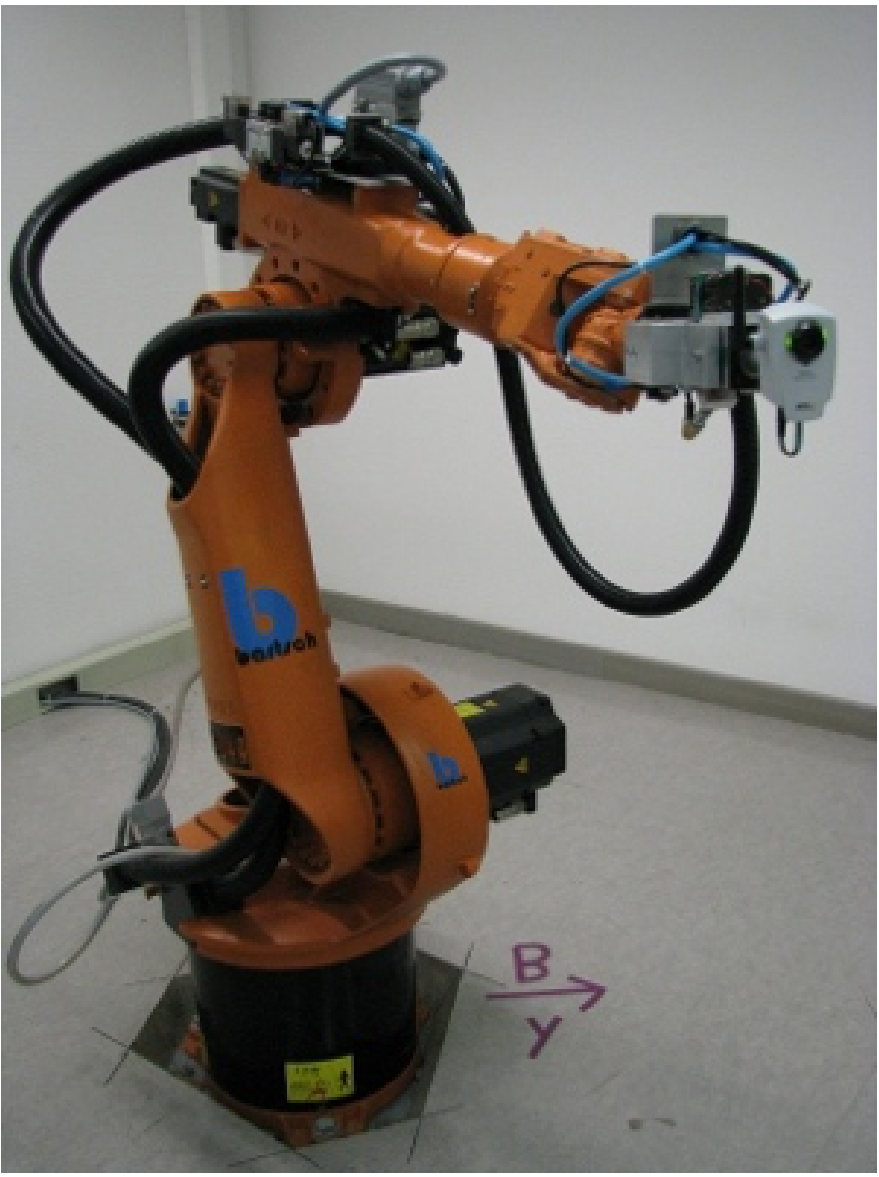

Fig. 4: The robot platform with the camera mounted in the gripper.

by any programming language with libraries supporting HTTP connections. Needless to say, the list of qualifying languages is extensive.

In order to transform the camera into a completely wireless component, a wireless power supply was developed. Namely, a custom voltage regulator was designed and fabricated to regulate the voltage of a battery pack down to the required $5 \mathrm{~V}$ for the camera. The regulator will operate with any DC voltage from 7 $25 \mathrm{~V}$, allowing interoperation with a wide variety of battery packs.

\subsection{Network}

In order to achieve our goal of portability, the network was designed around distributed components that use free and open source standards for interprocess communication. Each component in the network is capable of operating independently on its own machine from anywhere that has access to the central switch. In the case of our experiments, the central switch is a local 802.11 router providing WLAN access to the local computers in the laboratory. In our network setup, there are four components that share information across the LAN:

1) The KUKA robot computer running KRL programs and the OPC server

2) The Axis $207 \mathrm{MW}$ wireless network camera

3) The object reconstruction software

4) The external KUKA control software

The logical arrangement of these components, their interconnection, and the communication protocols used are illustrated

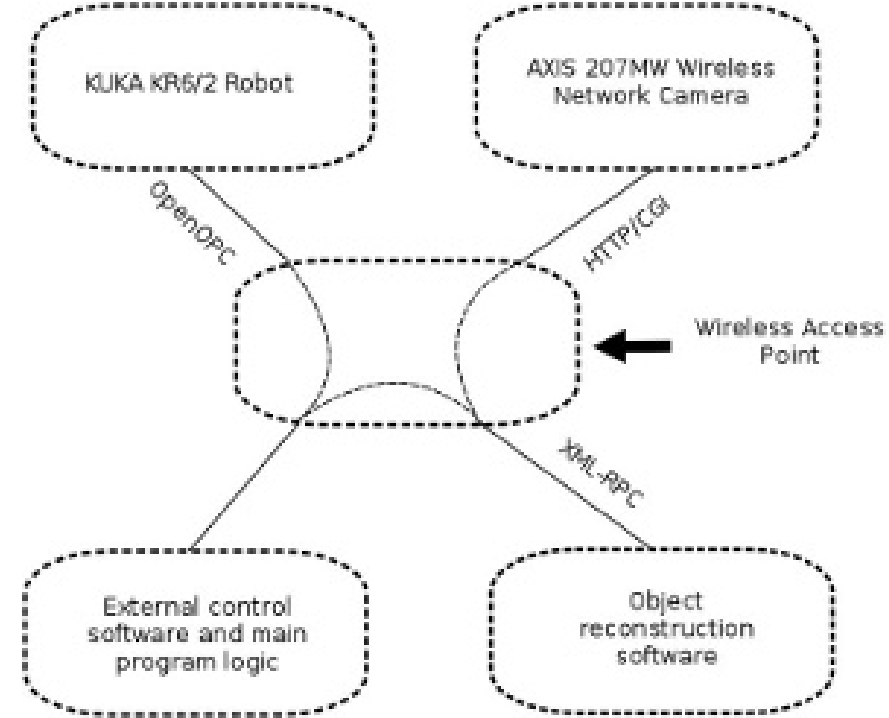

Fig. 5: Network and communication layout.

in following figure and are explained in detail in the following sections.

4.3.1 External KUKA Controller and the OPC Server: As previously mentioned, the KUKA robot software provides an OPC server that can be used to read and write system variables at run time. While OPC itself is an open standard, using it remotely requires extensive DCOM configuration which is both tedious and error prone, as well as limiting in that it requires the client machine to run a Microsoft Windows operating system. The OpenOPC project provides a solution to this problem. Built on Python, OpenOPC provides a platform agnostic method of making remote $\mathrm{OPC}$ requests. It runs a service on the host machine (in our case Windows XP embedded) which responds to requests from the client machine. The host service then proxies the OPC request to the (now local) OPC server, thus bypassing all DCOM related issues. The network communication transmits serialized Python objects ala the Pyro library.

A simple program was written in the KRL language and runs on the KUKA robot computer in parallel with the OPC server. This program sits in an idle loop monitoring the system variables until a command variable changes to True. At this point, the program breaks out of the loop and moves the robot to a position dictated by other system variables which are also set by the client machine. At the completion of the motion, the program re-enters the idle loop and the process repeats.

The external KUKA controller (the client) runs on a separate machine under Ubuntu Linux. This machine makes a connection to the OpenOPC service running on the KUKA computer and makes the appropriate requests to read and write the system variables. In this manner, this external machine is able to specify a desired robot position, either absolute or relative, and then, by setting the command variable to True, forces the robot to execute the motion. This machine also acts as the main control logic, synchronizing the robot motion with the image capturing and object reconstruction.

4.3.2 Wireless Camera and Object Reconstruction: The wireless camera presents itself on the network as an HTTP server where images can be obtained by making CGI requests. These requests are trivial to make using the Python urllib2 module. The data is received in the form of raw JPEG data which must be 


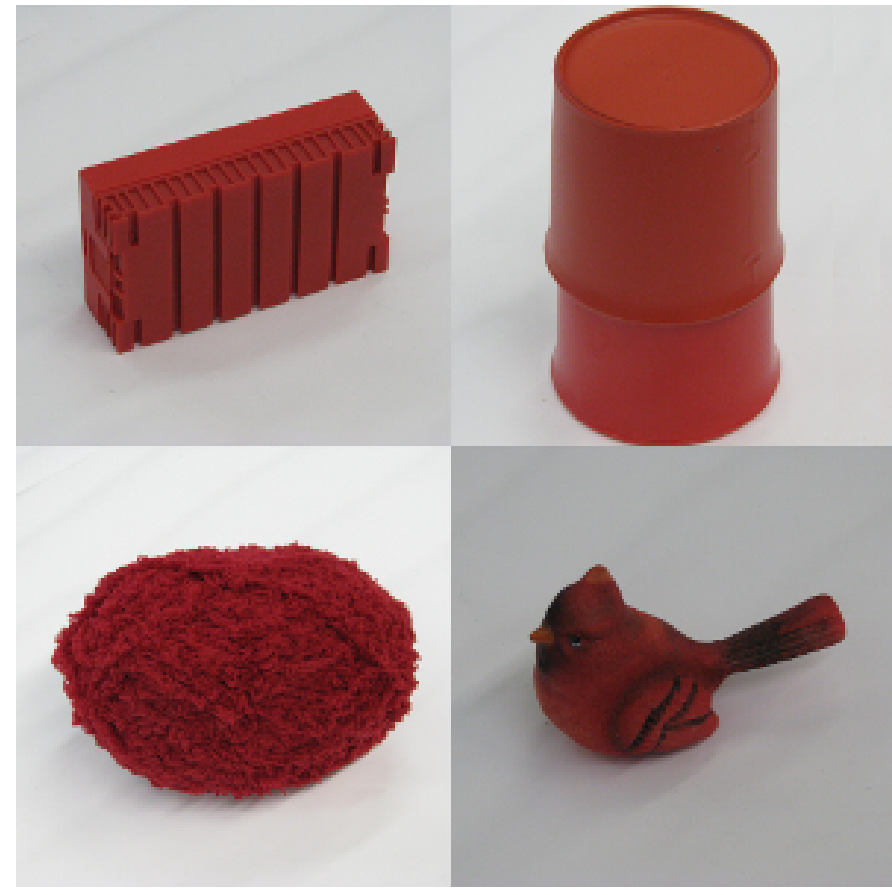

Fig. 6: The objects used for testing. Clockwise from upper-left: (1) A battery box. (2) A stack of cups. (3) A cardinal statue. (4) A ball of yarn.

converted to RGB raster format for purposes of image processing. This conversion is done using the Python Imaging Library. So that the data need not traverse the network twice, the connection to the camera is made from the object reconstruction program and images are captured and converted upon request by the main control program.

The connection between the main controller and object reconstruction program utilizes the XML-RPC protocol. The object reconstruction programs exports the majority of its capability in the form of methods on a SimpleXMLRPCServer instance from the Python xmlrpclib module.

\section{Testing and Results}

After verifying the accuracy of the algorithm in simulation, it was implemented on the hardware platform and tested on a variety of real world objects: a prismatic battery box, an elongated cylinder composed of two stacked cups, a ball of yarn, and a small cardinal statue. The first three objects represent the range of geometric shapes frequently encountered in domestic settings: cylindrical, prismatic, and ellipsoidal. It was expected that the algorithm would achieve accurate reconstructions for these shapes. The last object is amorphous and was included to test the robustness of the algorithm when presented with data that is incapable of being accurately described by the superquadric model. In all cases, the test objects were red in color to ease the task of segmentation and facilitate reliable silhouette generation. The four objects tested are shown in the following figure.

As seen previously in the simulated reconstruction, the recovered superquadric models the original object to high a degree of accuracy. On the real world objects, the accuracy of the algorithm was seen to degrade only slightly. Indeed, most parameters were recovered to within few percent of known ground truth. It must be kept in mind, however, that there are several sources of error that

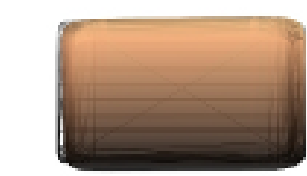

4

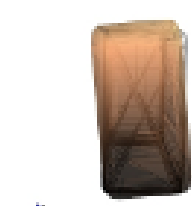

$\Delta$

*

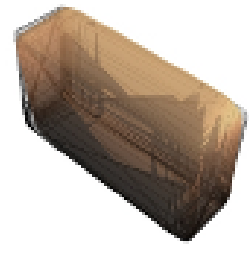

Fig. 7: The reconstruction of the battery box.

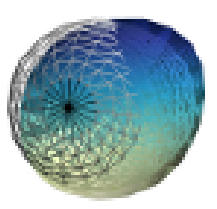

i.
Fig. 8: The reconstruction of the yarn ball.

are compounded into these reconstructions which are not present in the simulation:

- Uncertain camera calibration: intrinsics and extrinsics

- Robot kinematic uncertainty

- Imperfect segmentation

- Ground truth measurement uncertainty

The last bullet is particularly noteworthy. Since the object is placed randomly in the robot's workspace, the only practical way of measuring the ground truth position and orientation is to use a measuring device attached to the end effector of the robot. Though more accurate than attempting to manually measure from the robot base, the error is compounded by both machine inaccuracy and human error.

In the following figures, the results of the reconstruction for each of the cases is shown by a rendering of the known ground truth of the object accompanied by an overlay of the calculated superquadric. The ground truth is shown as a wire frame and the reconstruction as an opaque surface.

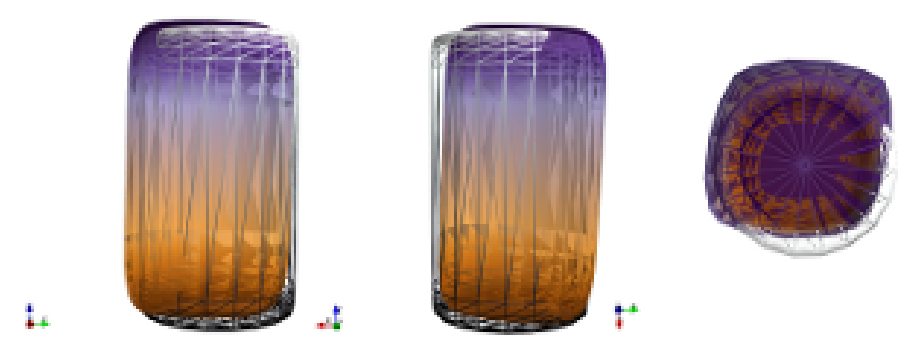

Fig. 9: The reconstruction of the cup stack.
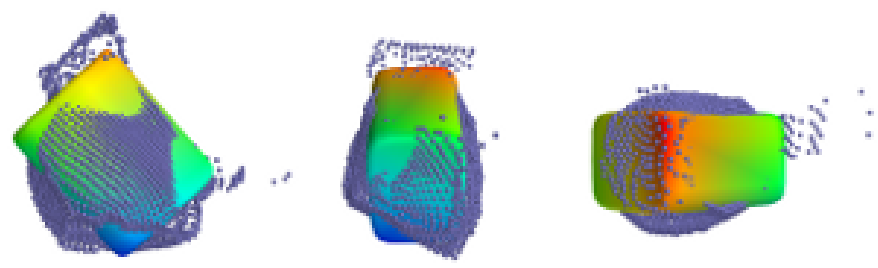

Fig. 10: The reconstruction of the cardinal statue. This original object is shown in terms of the computed point cloud, given the difficulty of modeling the amorphous shape as a wireframe. 
We feel that the results of the cardinal statue reconstruction are due a bit of explanation. We included this case to test how our algorithm performs when provided with data that does not fit well with our reconstruction model and assumptions, e.g. that the original object can be modeled well by a superquadric. From the figure, it is clear that there would be no way to infer from the box shape that is the final reconstruction that the original object was a cardinal figurine. However, it is interesting to note that the reconstruction is very close to what a human would likely provide if asked to select a bounding box that best describes the object. That is, the reconstructed shape does an excellent job of capturing the bulk form of the statue despite the fact that the data is ill formed with respect to our modeling assumptions.

This example shows that, even when the object does not take a form that can be accurately modeled by a single superquadric, our proposed algorithm still generates useful results.

\section{Conclusions}

We have given an overview of our robotic vision algorithm that is implemented in Python. Our algorithm enables the recovery of the shape, pose, position and orientation of unknown objects using just three images of the object. The reconstructions have sufficient accuracy to allow for the planning of grasping and manipulation maneuvers.

Both the algorithm and software side of the hardware implementation are implemented entirely in Python and related projects. Notable libraries used include: NumPy, SciPy, Cython, OpenOPC, and scikits.image. This software stack was proven to provide high performance with our algorithm executing in less time than other implementations in the literature.

\section{REFERENCES}

[Kim09] D. Kim, R. Lovelett, and A. Behal, Eye-in-Hand Stereo Visual Servoing on an Assistive Robot Arm in Unstructured Environments, International Conference on Robotics and Automation, pp. 2326-2331, May 2009.

[Effendi08] S. Effendi, R. Jarvis, and D. Suter, Robot Manipulation Grasping of Recognized Objects for Assistive Technology Support Using Stereo Vision, Australasion Conference on Robotics and Automation, 2008

[Schlemmer07] M. J. Schlemmer, G. Biegelbauer, and M. Vincze, Rethinking Robot Vision - Combining Shape and Appearance, International Journal of Advanced Robotic Systems, vol. 4, no. 3, pp. 259-270, 2007.

[Liefhebber07] F. Liefhebber and J. Sijs, Vision-based control of the Manus using SIFT, International Conference on Rehabilitation Robotics, June 2007.

[Kragic05] D. Kragic, M. Bjorkman, H. I. Christensen, and J. Eklundh, Vision for Robotic Object Manipulation in Domesting Settings, Robotics and Autonomous Systems, vol. 52, pp. 85-100, 2005.

[Colbert10_1] S. C. Colbert, R. Alqasemi, R. Dubey, Efficient Shape and Pose Recovery of Unknown Objects from Three Camera Views, International Symposium on Mechatronics and its Applications, April 2010.

[Colbert10_2] S. C. Colbert, R. Alqasemi, R. Dubey, G. Franz, K. Woellhaf, Development and Evaluation of a Vision Algorithm for $3 D$ Reconstruction of Novel Objects from Three Camera Views, IEEE International Conference on Intelligent Robots and Systems, 2010. in-press.

[Laurentini94] A. Laurentini, The Visual Hull Concept for Silhouette-Based Image Understanding, Transactions of Pattern Analysis and Machine Intelligence, vol. 16, Feb. 1994.

[Dyer01] C. R. Dyer, Volumetric Scene Reconstruction from Multiple Views, Foundations of Image Understanding, Boston: Kluwer, pp. 469-489, 2001.

[Lippiello09] V. Lippiello and F. Ruggiero, Surface Model Reconstruction of 3D Objects from Multiple Views, International Conference on Robotics and Automation, pp. 2400-2405, May 2009.
[Jaklic00]

A. Jaklic, A. Leonardis, and F. Solina, Segmentation and Recovery of Superquadrics, vol. 20 of Computational Imaging and Vision. Kluwer Academic Publishers, 2000.

[Yamazaki08] K. Yamazaki, M. Tomono, T. Tsubouchi, Picking up an Unknown Object Through Autonomous Modeling and Grasp Planning by a Mobile Manipulator, vol. 42/2008 of STAR. Springer Berlin / Heidelberg, 2008. 